\title{
Properties of Dense Fluid Hydrogen and Helium in Giant Gas Planets
}

\author{
Jan Vorberger*1, Isaac Tamblyn ${ }^{2}$, Stanimir A. Bonev ${ }^{2}$, and Burkhard Militzer ${ }^{1}$ \\ ${ }^{1}$ Geophysical Laboratory, Carnegie Institution of Washington, Washington D.C., USA \\ ${ }^{2}$ Department of Physics, Dalhousie University, Halifax, Nova Scotia, Canada
}

Received 03 November 2006

Key words density functional molecular dynamics, equation of state, giant gas planets, hydrogen-helium mixtures

PACS 61.20.Ja, 61.25.Em, 61.25.Mv, 61.20.-p

Equilibrium properties of hydrogen-helium mixtures under thermodynamic conditions found in the interior of giant gas planets are studied by means of density functional theory molecular dynamics simulations. Special emphasis is placed on the molecular-to-atomic transition in the fluid phase of hydrogen in the presence of helium. Helium has a substantial influence on the stability of hydrogen molecules. The molecular bond is strengthened and its length is shortened as a result of the increased localization of the electron charge around the helium atoms, which leads to more stable hydrogen molecules compared to pure hydrogen for the same thermodynamic conditions. The ab initio treatment of the mixture enables us to investigate the structure of the liquid and to discuss hydrogen-hydrogen, helium-helium, and hydrogen-helium correlations on the basis of pair correlation functions.

Copyright line will be provided by the publisher

\section{Introduction}

The discovery of the first extrasolar planet in 1995 [1] marked the beginning of a new era in planetary science, characterized by a rapidly expanding set of known extrasolar planets. More than 200 exoplanets have been discovered so far [2]. Among these, giant gas planets in small orbits are in the majority since the primary tool for detection, radio velocity measurements, is most sensitive to finding heavy planets that rapidly orbit their parent $\operatorname{star}[3,4]$.

From radius measurements of transient extrasolar planets, it is also known that most of these giant gas planets are like Jupiter in consisting primarily of hydrogen and helium. Modeling the interior of such planets requires an accurate equation of state for hydrogen-helium mixtures at high pressure and temperature conditions similar to those in planetary interiors [5]. Thus, the characterization of such system by first principle calculations will help us to answer questions concerning the inner structure of planets, their origin and evolution [5, 6].

In this article, we focus on studying the transition from molecular to atomic hydrogen. In particular, we investigate the effect of different helium concentrations on this transition. In what follows, it will be shown how the structure of the fluid and its equation of state (EOS) change with varying the hydrogen-helium mixing ratio.

\section{Method}

We use density functional molecular dynamics (DFT-MD) to investigate the questions connected with the physics of giant gas planets. Under conditions along Jupiter's isentrope, the electrons can be considered to be in their ground state. They are either chemically bound in molecules or atoms, or form a degenerate interacting electron gas. Density functional theory can thus be used to describe the electrons throughout. The ions form a strongly coupled fluid which makes many-body simulations an obvious choice for their description. For the interaction of the classical ions and electrons (quantum particles) the Born-Oppenheimer (BO) approximation can be invoked since the motions of electrons and ions follow different timescales.

\footnotetext{
* Corresponding author: e-mail: jvorberger@ciw.edu, Phone: +001 202478 8943, Fax: +001 2024788901
} 


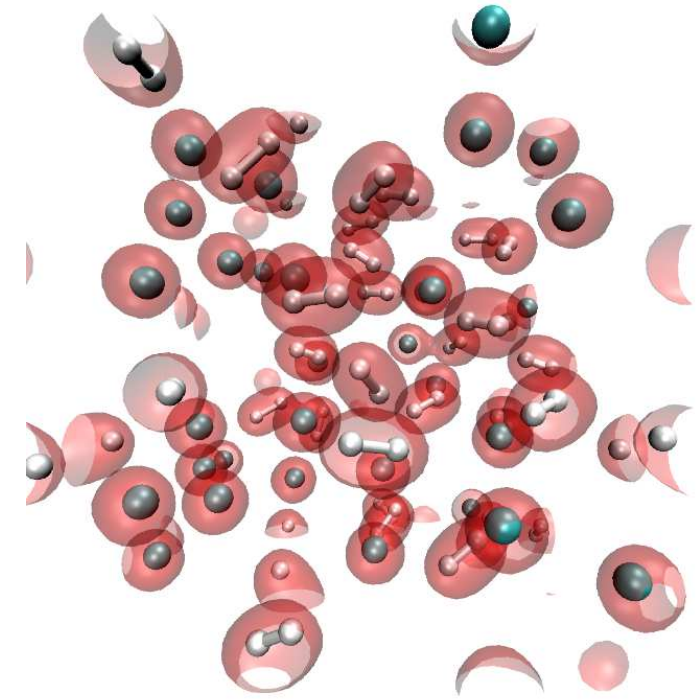

a)

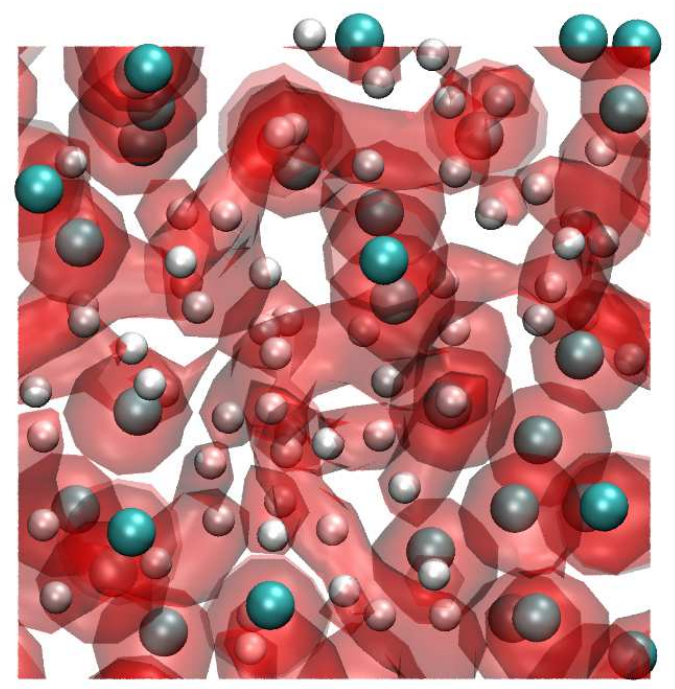

b)

Fig. 1 View of the simulation cell of a DFT-MD simulation. Hydrogen and helium nuclei are represented by small and large spheres, respectively, and electron density isosurfaces are drawn. In these plots, the mixing ratio is $x=0.5$, and the temperature is $4000 \mathrm{~K}$. The density corresponds to a) $r_{s}=2.4\left(0.3 \mathrm{~g} / \mathrm{cm}^{3}\right)$ at pressure of $8 \mathrm{GPa}$, and b) $r_{s}=0.9\left(5.5 \mathrm{~g} / \mathrm{cm}^{3}\right)$ at pressure of $3800 \mathrm{GPa}$. These conditions correspond to a molecular phase and to a metallic regime of fluid hydrogen, respectively.

The results presented here are obtained using the CPMD code [7]. We use simulations cells with 128 electrons and the corresponding number of ions, and periodic boundary conditions. The forces acting on the ions are provided by DFT calculations within generalized gradient approximation (GGA) for the electrons in the Coulomb field of the ions. We use Troullier Martin norm-conserving pseudopotentials for the electron-ion interactions [9]. To check for finite-size effects, calculations with supercells ranging from 54 to 250 atoms are carried out, but give no significant change in the results $(<2 \%$ in pressure for supercells with 128 or more particles). We have also performed simulations with different number of $\mathrm{k}$ points (1 to 512) to sample the Brillouin zone, and it was confirmed that a single $\mathrm{k}$ point already gives converged results for the supercells and conditions used here. Recently, it was shown that in high pressure water effects arising from the treatment of the electrons at their true temperature instead of in the ground state can be important for the dynamics of the system [10]. Therefore, special attention was given to the effects arising from a finite electron temperature. We have performed additional DFT-MD simulations with the VASP code using the finite temperature Mermin functional to model the influence of thermal occupation of electronic states [11]. For Jupiter's mixing ratio of $x=N_{H e} /\left(2 N_{H}+N_{H e}\right)=0.14$ almost no difference could be detected at relevant temperatures in the metallic regime (less than half a percent deviation).

\section{Equation of State and Structure of the Hydrogen-Helium Fluid}

Figure 1 displays snapshots of the simulation cell during runs for two different situations. In Fig. 1h) the molecular fluid at low density $\left(r_{s}=2.4\right)$ is characterized by larger distances between the molecules and by electronic densities concentrated around the nuclei. Such a molecular fluid can be found in the outer mantle regions of Jupiter. The physical system reminiscent to the inner mantle of Jupiter, shown in Fig. 1b), can be characterized as a metallic fluid. The density is much higher, molecules are dissociated as a result of the increased pressure. The electrons are delocalized as a result of the Pauli exclusion principle and form an electron cloud that extends through the simulation cell.

For given temperature and density conditions, which of the two described phases can be found, namely, molecular or atomic/metallic, depends strongly on the helium concentration. Moreover, the helium fraction plays a 


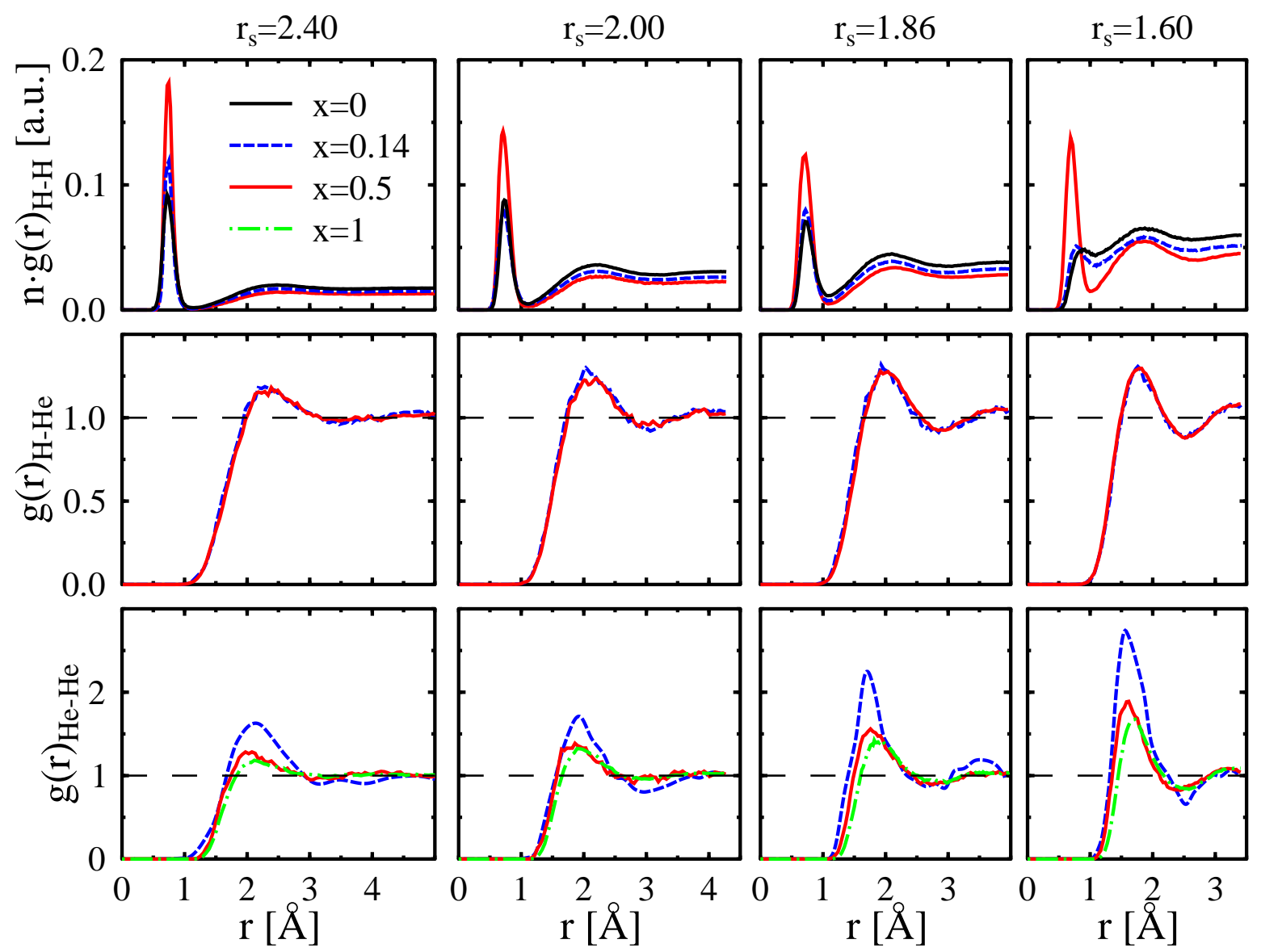

Fig. 2 Pair correlation functions at a temperature of $3500 \mathrm{~K}$ across the molecular-atomic transition for various densities and mixing ratios. The three rows of graphs show the hydrogen-hydrogen, hydrogen-helium, and helium-helium pair correlation functions. Here, $g(r)_{H-H}$ was multiplied by the concentration of hydrogen atoms so that the area under the peak at $r=$ 0.75 A corresponds to the fraction of molecules in the fluid. In each graph, simulations for different mixing ratios have been combined: pure hydrogen $(x=0)$, hydrogen and helium for Jupiter's mixing ratio $(x=0.14)$, a fluid with as many helium atoms as hydrogen molecules $(x=0.5)$, and pure helium $(x=1)$. The columns show results from different electronic densities (given in terms of the Wigner Seitz parameter $r_{s}$ ), for which different mixing ratios have been compared.

crucial role in how the mixture transforms from the molecular to the atomic phase with increasing temperature or pressure.

Changes in the structure of the fluid, i.e., the transition from a molecular to a metallic fluid may be discussed in terms of the pair correlation function $(\mathrm{PCF})$

$$
g(r)=\frac{V}{N(N-1)}\left\langle\sum_{i \neq j} \delta\left(r-\left|r_{i j}\right|\right)\right\rangle \frac{1}{4 \pi r^{2}} .
$$

Pair correlation functions for different types of nuclei are shown in Fig. 2. We discuss the changes in the pair correlations with respect to density at a constant temperature of $3500 \mathrm{~K}$. Let us first comment on the hydrogenhydrogen pair correlations in pure hydrogen. A pronounced peak at about $r=0.75 \AA$ demonstrates the presence of molecules in the fluid. These pair correlations are normalized so that the area under the molecular peak gives the number of molecules found in the fluid. The dissociation degree can thus be extracted either by integrating over the PCF or by invoking a distance criterion for two hydrogen atoms. Table 1 reports degrees of dissociation that where derived by analyzing the MD trajectories using the distance criterion and a minimum molecular 


\begin{tabular}{c||c|c|c|c} 
& $r_{s}=2.40$ & $r_{s}=2.00$ & $r_{s}=1.86$ & $r_{s}=1.60$ \\
\hline \hline \multirow{2}{*}{$\mathrm{x}=0.0$} & $8.9 \pm 0.1 \mathrm{GPa}$ & $25.5 \pm 0.1 \mathrm{GPa}$ & $38.5 \pm 0.3 \mathrm{GPa}$ & $89.4 \pm 0.2 \mathrm{GPa}$ \\
& 0.94 & 0.38 & 0.20 & 0.05 \\
\hline \multirow{2}{*}{$\mathrm{x}=0.14$} & $8.5 \pm 0.1 \mathrm{GPa}$ & $24.1 \pm 0.2 \mathrm{GPa}$ & $38.4 \pm 0.2 \mathrm{GPa}$ & $90.8 \pm 0.3 \mathrm{GPa}$ \\
& 0.98 & 0.38 & 0.43 & 0.09 \\
\hline \multirow{2}{*}{$\mathrm{x}=0.5$} & $7.0 \pm 0.1 \mathrm{GPa}$ & $21.2 \pm 0.1 \mathrm{GPa}$ & $33.0 \pm 0.2 \mathrm{GPa}$ & $89.7 \pm 0.2 \mathrm{GPa}$ \\
& 0.97 & 0.45 & 0.44 & 0.49 \\
\hline \multirow{2}{*}{$\mathrm{x}=1.0$} & $5.3 \pm 0.1 \mathrm{GPa}$ & $14.7 \pm 0.1 \mathrm{GPa}$ & $22.5 \pm 0.1 \mathrm{GPa}$ & $65.0 \pm 0.1 \mathrm{GPa}$ \\
& - & - & - & -
\end{tabular}

Table 1 Values for pressure (1st line) and fraction of atoms bound in molecules (2nd line) at various densities and mixing ratios at $T=3500 \mathrm{~K}$. These values belong to the pair correlation functions in Fig. 2 .

lifetime of 10 vibrational periods of the isolated hydrogen molecule. The lifetime criteria leads to a decaying molecular fraction in the metallic regime, which would be overestimated based on the distance criterion alone.

A high peak followed by a minimum that approaches zero is a strong indication that the molecules are stable, for instance this is the case at $r_{s}=2.4$ for different values of $x$. Indeed, this can be confirmed by directly analyzing the number and lifetime of the molecules [14]. On the other hand, at high density $\left(r_{s}=1.6, x=0\right)$, no isolated first peak can be identified and the shape of the PCF is typical for a correlated atomic liquid. The first row of Fig. 2 contains two additional graphs showing the pair correlations at densities and mixing ratios intermediate between the two extrema just discussed. In these cases, the molecular peak can still be identified but its height is reduced and it is widened so that the first minimum has a value greater than zero. A shape like this is caused by a mixture of atoms and molecules; where the definition of molecules here is somewhat ambiguous as they may have finite lifetimes.

Let us now discuss the influence of helium on the PCF. The general effect can be seen by comparing hydrogenhydrogen pair correlation functions at identical electronic densities and different helium fractions. More helium content in the mixture generally leads to a lower hydrogen dissociation. The presence of helium atoms thus stabilizes the hydrogen molecules and the effect of pressure to dissociate hydrogen molecules is partially suppressed. A more detailed analysis revealed that in mixtures of hydrogen and helium the bond length of the hydrogen molecule is shortened by about $6 \%$ [12, 14]. The influence of helium allows hydrogen molecules to exist in a parameter regime where otherwise pure hydrogen would be dissociated. The microscopic reason for this effect is the double charge of the helium nuclei binding two electrons. These electrons are bound stronger than the electrons in a hydrogen molecule and are more localized, which has profound effects on the high pressure properties of the fluid [13]. In the mixture, atoms and molecules can be packed more closely before electronic wavefunctions begin to overlap. When molecules are compressed and wave functions begin to overlap, their localization increases because of the exclusion principle, which means larger kinetic energy. But the kinetic and potential energies scale differently with density, so eventually, it is energetically more favorable for the electrons to delocalize (lower kinetic energy, higher potential energy).

We also examine helium-helium (He-He) and hydrogen-helium (H-He) pair correlation functions extracted from the DFT-MD simulations. Both, He-He and H-He pair correlation functions show a finite probability for finding a neighboring atom only for distances larger than $1.5 \AA$. This value reflects the bigger radius of a helium atom compared to a hydrogen atom.

Hydrogen-helium correlations are similar to the continuous part of the hydrogen-hydrogen PCFs. Naturally, there is no molecular peak and significant contributions occur at distances larger than the first minimum of the hydrogen-hydrogen PCFs. Thereafter, similar weak short-range order expressed in oscillations around unity can be observed. There are only minor differences between hydrogen-helium correlation functions of different mixing ratios.

The correlations in the helium subsystem can be described as short-range hard sphere type interactions. Interestingly, the helium-helium pair correlations show a more pronounced first peak in the $x=0.14$ mixture than in the $x=0.5$ mixture and they again feature a slightly higher peak than the ones for pure helium. There is a bigger total number of hydrogen atoms present for a lower mixing ratio $x$ at constant electronic density. Two 

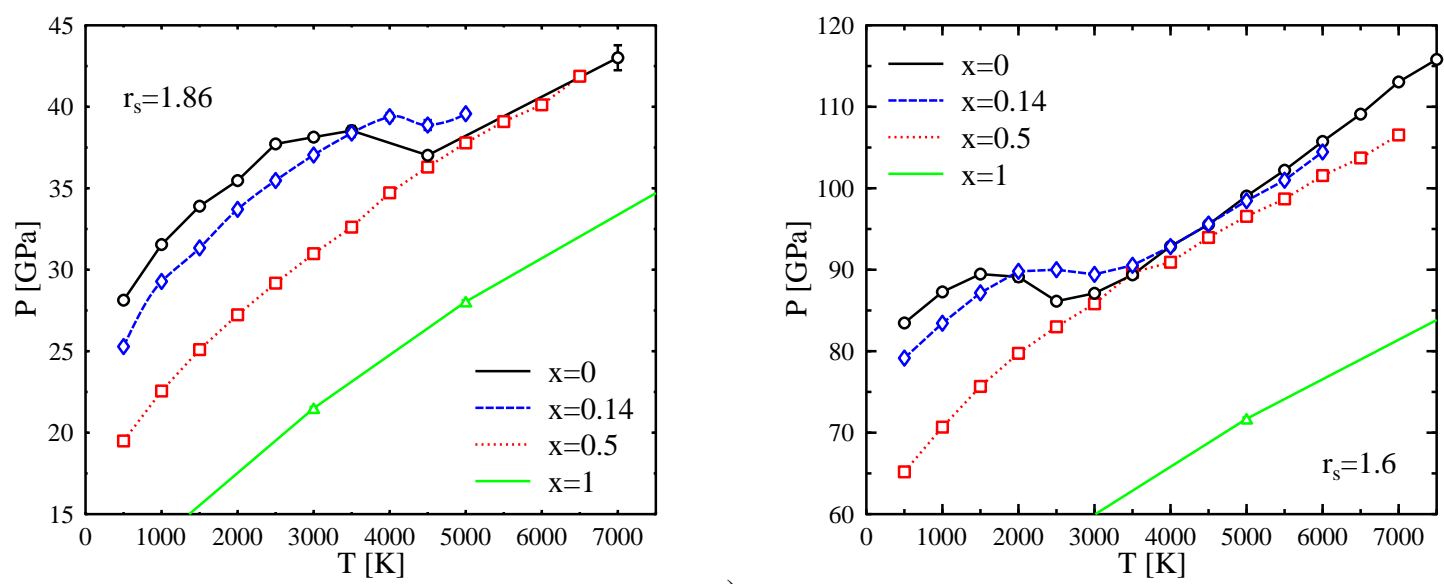

a)

b)

Fig. 3 Pressure vs. temperature isochores for hydrogen, hydrogen-helium mixtures of various mixing ratios $x$, and helium for two different electronic density parameters $r_{s}$ : a) $r_{s}=1.86$ corresponds to $\rho=0.42 \mathrm{~g} / \mathrm{cm}^{3}$ in hydrogen, and b) $r_{s}=1.6$ corresponds to $\rho=0.67 \mathrm{~g} / \mathrm{cm}^{3}$ in hydrogen.

hydrogen atoms or one hydrogen molecule have a bigger volume than one helium atom. The effective density for the helium atoms is thus higher in a mixture with less helium. This causes a general requirement to increased coordination among the atoms which in turn is reflected in the higher peak in the pair correlation. The effect of hydrogen on helium can be understood as the opposite of the effect of helium on hydrogen.

The observed structure in helium-helium and hydrogen-helium pair correlations illustrates the importance of the interaction between the subsystems and is an indication that the linear mixing rule is only a first approximation for describing mixtures [14].

Naturally, changes in the structure of the fluid have an effect on the equation of state (EOS) as presented in Fig. 3. The pressure isochores show some distinct features depending on the helium concentration. Since helium atoms are smaller than hydrogen molecules, one finds that at low temperature the pressure decreases substantially when hydrogen molecules are replaced by helium atoms at constant volume, which leaves the total electronic density unchanged. In pure hydrogen, the isochores exhibit a region with a negative slope $d P /\left.d T\right|_{V}<0$, which is due to the delocalization of the electrons during the dissociation of hydrogen molecules. A higher helium fraction reduces the magnitude of the drop in pressure (e.g. for $x=0.14$ ) or even completely removes it $(x=0.5)$. As a result, one finds that the $P(T)$ curves for $x=0, x=0.14$, and $x=0.5$ approximately converge in the dissociated regime. There, the pressure depends only little on the helium concentration because the (ideal) ionic pressure increase and the pressure decrease due to electronic delocalization depend inversely on the mixing ratio. Only for large helium concentration this cancellation effect does not occur anymore as the comparison with the $x=1$ curve shows.

Fig. 3 alone demonstrates temperature dissociation. By comparing the left and the right part of Fig. 3, it is possible to observe pressure dissociation, too. The region with negative slopes indicating molecular dissociation is shifted to smaller temperatures for higher densities. This means that, by increasing the density at constant temperature, one can transfer from a molecular fluid to an atomic fluid as well.

\section{Conclusion}

The structure of the fluid and the equation of state for hydrogen-helium mixtures were analyzed under conditions found in giant gas planets. We have considered the structural changes in the fluid during dissociation as function of density and helium concentration. DFT-MD proves to be an useful technique to study such questions and to obtain accurate equation of state data. Such data are needed in order to improve existing models for giant gas planets and answer longstanding question concerning their development and inner structure. 
In particular, we find that helium plays a crucial role in affecting hydrogen dissociation both as a function of temperature and pressure. Hydrogen molecules are stable at higher temperatures and pressures in mixtures of hydrogen and helium than in pure hydrogen. The transition from the molecular to the atomic phase proceeds over a wider range of temperature and pressure. We observe regions with negative slope of the pressure isochores in pure hydrogen as well as in hydrogen-helium mixtures, but only up to a critical mixing ratio.

Acknowledgements This material is based upon work supported by NASA under the grant NNG05GH29G, by the Carnegie Institution of Canada, and by the NSF under the grant 0507321. I.T. and S.A.B. acknowledge support by the NSERC of Canada.

\section{References}

[1] M. Mayor and D. Queloz. Nature, 378:355, 1995.

[2] The extrasolar planet encyclopedia. http://exoplanet.eu

[3] A. Burrows. Nature, 433:261, 2005.

[4] Rivera et al., APJ 634:625, 2005.

[5] T. Guillot el al., in Jupiter, F. Bagenal, Ed. (Univ. of Arizona Press, Tucson, 2003), chap. 3, pp. 35-57.

[6] D. Saumon and T. Guillot. Astrophys. J., 609:1170, 2004.

[7] CPMD, version 3.9.2, Copyright IBM Corp 1990-2006, MPI für Festkörperforschung Stuttgart 1997-2001.

[8] J. P. Perdew, K. Burke, and M. Ernzerhof. Phys. Rev. Lett., 77:3865, 1996.

[9] N. Troullier and J. L. Martin. Phys. Rev. B, 43:1993, 1001.

[10] T. Mattson and M.P. Desjarlais Phys. Rev. Lett., 97:2006, 017801.

[11] G. Kresse and J. Hafner. Phys. Rev. B, 47:558, 1993, G. Kresse and J. Hafner. Phys. Rev. B, 49:14251, 1994, G. Kresse and J. Furthmüller. Comput. Mat. Sci., 6:15, 1996, G. Kresse and J. Furthmüller. Phys. Rev. B, 54:11169, 1996.

[12] O. Pfaffenzeller, D. Hohl, and P. Ballone. Phys. Rev. Lett., 74:2599, 1995.

[13] B. Militzer Phys. Rev. Lett, 97:175501, 2006.

[14] J. Vorberger, I. Tamblyn, B. Militzer, S.A. Bonev submitted to Phys. Rev. B, 2006, cond-mat/0609476 